\title{
Design and Comparative Analysis of SVM and PR Neural Network for Classification of Brain Tumour in MRI
}

\author{
Meenu Rohilla \\ DCRUST Murthal (ECE Deptt.) \\ Hindu College of Engineering \\ DCRUST Murthal, India
}

\author{
Vinay Singal \\ Asst. Prof. (ECE Deptt.) \\ Hindu College of Engineering \\ DCRUST Murthal,India.
}

\begin{abstract}
Electroencephalograms (EEGs) or MRI are progressively emerging as a significant measure of brain activity and they possess immense potential for the diagnosis and treatment of mental and brain diseases and abnormalities. This research paper presents an automated system for efficient classification of brain tumours in MRI images using Support Vector Machine (SVM). It works on the principle of a vector which supports in training of classifier using train images. The results obtained from the above method are compared with those obtained from Artificial Neural Networks (ANNs). This method uses pattern recognition algorithms for the classification of tumour in MR images. First, a pattern recognition neural network is created and then trained. The trained pattern recognition neural network when fed with a test MR image, effectively classifies the images. The performance analysis shows that qualitative results obtained from the proposed model are comparable with those obtained by ANN. The experimental results demonstrate the effectiveness of the proposed system in classification of brain tumour in MR images. The implementation is done using MATLAB R2012b using Artificial Neural Network and Image Processing Toolbox.
\end{abstract}

Keywords - Tumour, MRI, Pattern recognition, SVM, Neural network.

\section{INTRODUCTION}

For years now, the application of EEG in neurosurgery has been focused on the identification of brain lesions. EEG recorded by the scalp electrodes is a superposition of an enormous amount of electrical potentials produced from a number of sources (including brain cells i.e. neurons and artefacts). Among the artefacts present, the Electro-oculogram (EOG) signal is the principal and most prevalent artefact in EEG analysis, generated by eye movements and/or blinks. In recent times, it has become tremendously simple to proficiently eradicate the eye-blink artefacts without affecting the underlying brain activity. A better solution would be to determine the signals of interest from the EEG recorded from the scalp using suitable signal processing techniques. The pattern of electrical activity obtained from the EEG signal is chiefly valuable as well utilized to examine other conditions that might affect brain function, like head injuries, brain tumours or bleeding on the brain.

\subsection{Existing Methods}

A large number of techniques have been developed for medical image analysis in the last decade. In this section, some of the significant

approaches proposed by the researchers have been presented. One of the most used method in Tumour detection is by using image segmentation [2]. Image segmentation is the task of dividing an image into homogeneous regions. Two types of learning methods have been used in image segmentation, viz, unsupervised and supervised methods. The difference between Supervised and Unsupervised methods is that supervised methods make use of training data that has been manually labelled, while unsupervised methods do not. The advantage of supervised methods is that relevant patterns in the data are discovered automatically, rather than through manual experimentation and intuition [2]. Another mostly used existing method is based on the thresholding and region growing. In the thresholding based segmentation the image is considered as having only two values either black or white. But the bit map image contains 0 to 255 gray scale values. So sometimes it ignores the tumour cells also. In case of the region growing based segmentation it needs more user interaction for the selection of the seed. Seed is nothing but the centre of the tumour cells; it may cause intensity in homogeneity problem. And also it will not provide the acceptable result for all the images. The typical output for the thresholding is given below.
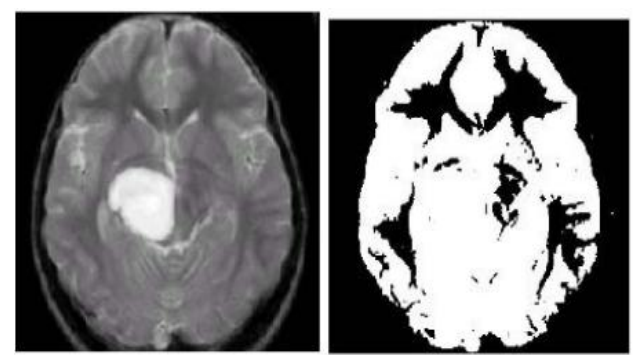

Fig 1: Input image for Thresohlding and output Image for Thresohlding [5]

Fig. 1 is the input image for thresholding. From the MR image the tumour area can be seen but it is not enough for further treatment. For that it is given to the threholding process. Fig. 2 is the output image for the thresholding. It consists of only two gray values .That is white as 1 and black as 0 . The background value is assigned to binary value 0 and object gets 
the value 1 . So tumour can't be extracted from the image. This is the main drawback of the existing system. Due to that there is a need to go for the proposed method for tumour segmentation.

The purpose of this project is to classify the brain tissues to normal and abnormal classes automatically, that saves the radiologist time, increases accuracy and yield of diagnosis.

\subsection{Support Vector Machine (SVM)}

Support vector machines are a particular family of learning machines, as an alternative to neural networks. SVM are classifiers with the distinct characteristic that they aim to find the optimal hyper-plane such that the expected generalization error (i.e., error for the unseen test patterns) is minimized. Instead of directly minimizing the empirical risk calculated from the training data, SVMs perform structural risk minimization to achieve good generalization (i.e., minimize an upper bound on expected generalization error). The optimization criterion is the width of the margin between the classes (i.e., the empty area around the decision boundary defined by the distance to the nearest training patterns). The goal of training a SVM is to find the separating plane with the largest margin (i.e., find the support vectors).

In the case of support vector machines, a data point is viewed as a p-dimensional vector (a list of $\mathrm{p}$ numbers), and it must be known whether such points with a $\mathrm{p}-1$ dimensional hyperplane can be separated. This is called a linear classifier. The model used for comparison makes use of a polynomial kernel represented by,

$$
K\left(x, x^{\prime}\right)=\left(1+\left(x^{T} x^{\prime}\right)\right)^{p}
$$

Where $p=2,3, \ldots$ To get the feature vectors, concatenation of all $\mathrm{p}^{\text {th }}$ order polynomial terms of the components of $\mathrm{x}$ (weighted appropriately) must be done. The rationale behind using the polynomial kernel of degree 3 is that the previous experiments have shown that SVM with a linear kernel obtained the best classification score $\mathrm{b} / \mathrm{w}$

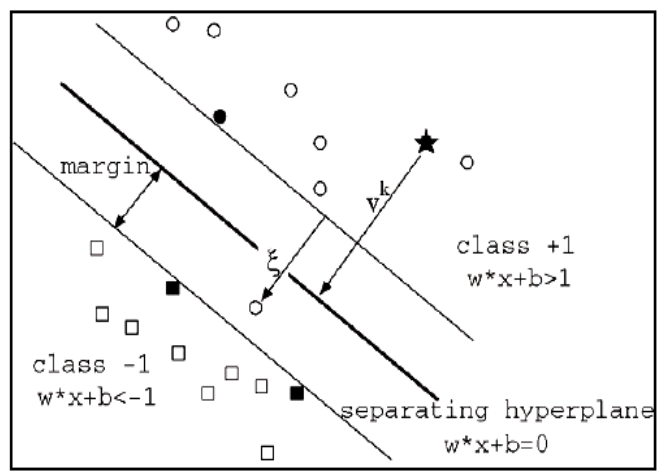

Fig 2: Projection of Data Points at hyper-plane in Linear SVM

$80-85 \%$, whereas SVM with polynomial kernel of degree 3 obtained the best classification score $88-90 \%$.

\subsection{Artificial Neural Network}

An Artificial Neural Network (ANN) is an information processing paradigm that is inspired by the way biological nervous systems, such as the brain, process information. The key element of this paradigm is the novel structure of the information processing system. It is composed of a large number of highly interconnected processing

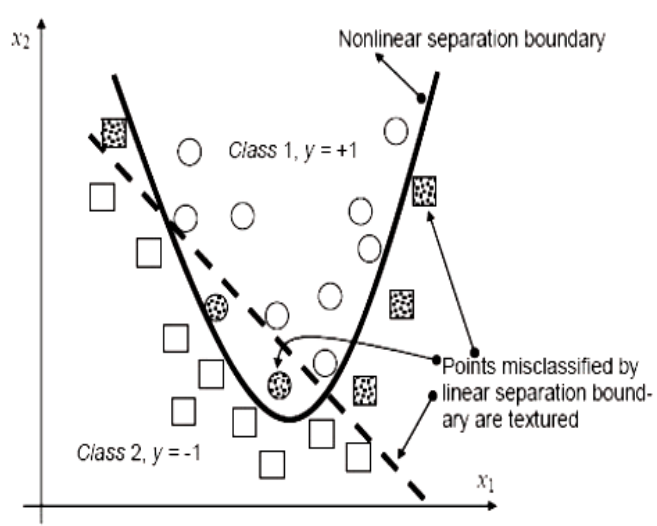

Projection of Data Points at hyper-plane in Non linear SVM

elements (neurons) working in unison to solve specific problems [4]. An ANN is configured for a specific application, such as pattern recognition or data classification, through a learning process. ANNs, like people, learn by example. Neural networks, with their remarkable ability to derive meaning from complicated or imprecise data, can be used to extract patterns and detect trends that are too complex to be noticed by either humans or other computer techniques. A trained neural network can be thought of as an "expert" in the category of information it has been given to analyze.

However, some network capabilities may be retained even with major network damage. An artificial neuron is a device with many inputs and one output. The neuron has two modes of operation; the training mode and the using mode. In the training mode, the neuron can be trained to fire (or not), for particular input patterns. In the using mode, when a taught input pattern is detected at the input, its associated output becomes the current output.

Artificial neural networks (ANN) are one of the most common classifiers used in MR image processing. Multilayer perceptron neural networks have been used to classify the Patterns extracted from the spectra. This method Pattern recognition neural network extracted the Patterns related to metabolite peaks and the second one extracted several features expressing the whole signal characteristics. This class of patterns is used in various sets to find the best features describing each lesion. In order to train the network and verify its ability to distinguish between different tumour spectral features, available data into two sets have been divided. The first set was used for training and the remaining set was used for testing [4].

\subsection{Pre-processing}

Pre-processing of brain MR image is the first step in proposed technique. Pre-processing of an image is done to reduce the noise and to enhance the brain MR image for further processing.

A random sample of diseased brain MR image is shown in Figure 4. The white abnormal mass accumulation shows the tumour area. By applying the adaptive median filter with 2 dimensions and a block size of [5,5], Figure 4 is enhanced and it is shown in other half part of it. The noise is reduced and the image is visibly smoothened. 


\section{PROPOSED METHODOLOGY}

\subsection{For SVM}

1. Calling of train images

2. Reading of image one by one

3. Changing its format into double type

4. Conversion into gray scale

5. Application of median filter on received image

6. Resizing and Reshaping of image

7. Generation of train data

8. Embedding of image data into train data

9. Construction of a column vector array of numbers ( 1 for normal image and -1 for tumour) for classifying data in Train Data into two groups. It has the same number of elements as there are rows in Train data. Each element specifies the group to which the corresponding row in Train data belongs.

10. Training of a support vector machine classifier using Train data.

11. Calling of test images

12. Reading of image one by one

13. Changing its format into double type
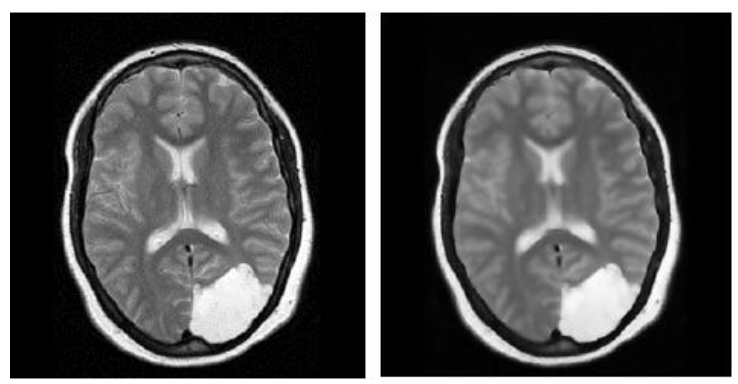

Fig 4: Raw data image and Adaptive median

14. Conversion into gray scale

15. Application of median filter on received image

16. Resizing and Reshaping of image

17. Classification of data using a support vector machine (so as to get result vector) by classify each row of the data in test image using the information in a support vector machine classifier.

18. Examination of result vector so as to distinguish normal images and images with tumour ( 1 for normal image and 1 for tumour).

19. Calculation of the incorrect and correct classification percentage of classifier.

\subsection{For Pattern Recognition Neural Network}

1. Calling of train images

2. Reading of image one by one

3. Changing its format into double type.
4. Conversion into gray scale.

5. Application of median filter on received image.

6. Resizing and Reshaping of image into a row vector.

7. Formation of input matrix by putting row vector of each test image one by one.

8. Conversion of input matrix into simple class input by platform (MATLAB Neural network Tool) itself.

9. Generation of a target matrix (default $=0$ or 1 ) i.e. either one or zero for each row of test matrix.

10. Conversion of target matrix into simple class target by platform (MATLAB Neural network Tool) itself.

11. Selection of appropriate number of neurons.

12. Generation of new Pattern Recognition Neural Network with the help of number of neurons, target matrix and input matrix.

13. Selection of proper network parameter i.e. validation ratio, test ratio and training ratio.

14. Training of network using input and target matrix so as to get a new trained network and training record.

15. Simulation of the network with the help of simple class input matrix so as to get the output matrix.

16. Plotting of confusion matrix with the help of output matrix and simple class target matrix so as to evaluate the performance of network classifier.

17. Calculation of the incorrect and correct classification percentage of classifier.

\section{RESULTS}

Two techniques i.e. SVM and PR neural network have been designed and examined for experimental purpose. This section describes the results of both techniques in terms of plots and snapshots. All experiments have been performed on a computer with $2.3 \mathrm{GHz}$ Intel Core $\mathrm{I} 3$ processor (CPU) and 2 GB main memory. The operating system of the computer is Microsoft Windows 7. All the algorithms have been implemented in MATLAB (R2012b). In this paper, experiments have been performed on brain MRI data set. Some of the test images which were considered for this experiment are shown in Figure $5-10$. Firstly, the case of classification of input test images through SVM is taken. The correct classification percentage is 95.3 and incorrect classification is 4.7 as shown in figure 12 whereas the figure 11 shows the Bar graph of test images. Higher limit peaks (up to 3 ) indicates the presence of tumour and lower limit peaks (up to 1) indicates the absence of tumour. Next is the case of PR using neural network which is working as a classifier for input test images. The correct classification percentage is 58.33333 and incorrect classification is 41.66777 , whereas next figures 13 and 14 show the confusion matrix and performance plot for this classifier. Confusion plot (figure 13) is another measure of how well the neural network has fit the data. Here the confusion matrix is plotted across all samples. The confusion matrix shows the percentages of correct and incorrect classifications. Correct classifications are the green squares on the matrices diagonal. Incorrect classifications form the red squares. If the network has learned 
to classify properly, the percentages in the red squares should be very small, indicating few misclassifications. Performance is measure of network's performance improved during training in terms of mean squared error, and shown in figure 14 in log scale. It rapidly decreased as the network was trained. Performance is shown for each of the training validation and test sets. The version of the network that did best on the validation set is was after training. The mean squared error of the trained neural network can now be measured with respect to the testing samples. Table 1 shows the correct and incorrect percentages of classification.

\section{CONCLUSION}

A modified version of SVM based algorithm is proposed for the classification of MRI images. The performance analysis shows that qualitative results obtained from the proposed model are comparable with those obtained by existing algorithm. However, in order to obtain better results from the proposed algorithm, there is a need to carefully select the different values of input parameters. So, it can be concluded easily by comparing the correct and incorrect classification percentage and other performance aspects of both techniques that SVM is much better technique than PR using neural network. This work can be extended for future work by increasing correct classification percentage for a set of MR images by selecting suitable input parameters.

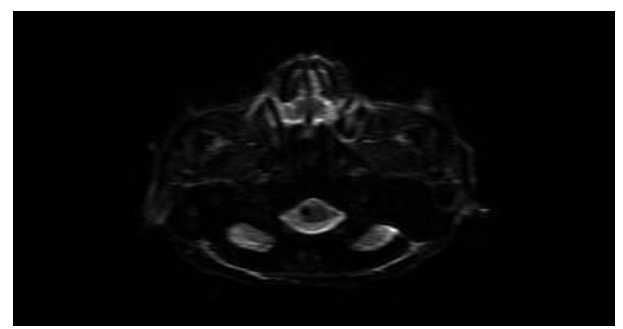

Fig 5: Test image 1

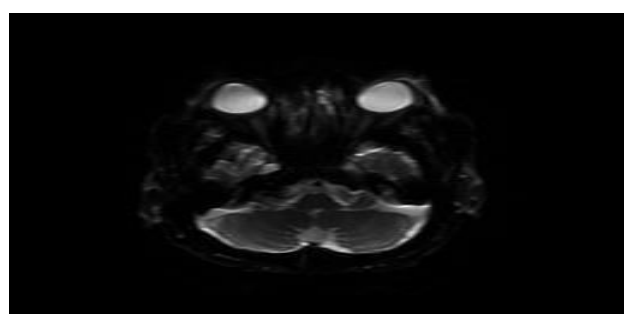

Fig 6: Test image 2

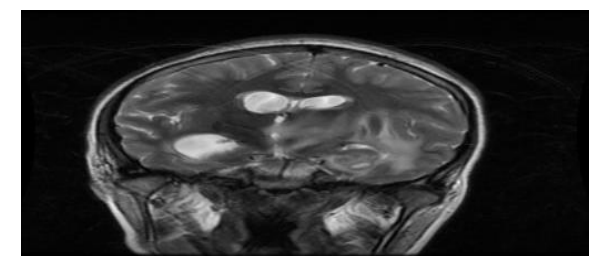

Fig 7: Test image 3

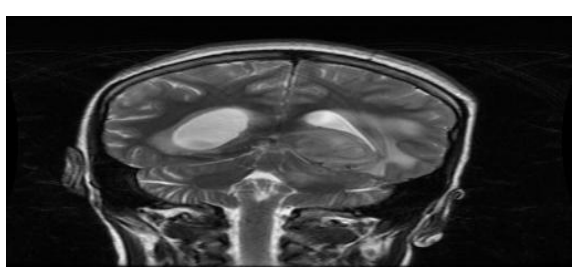

Fig 8: Test image 4

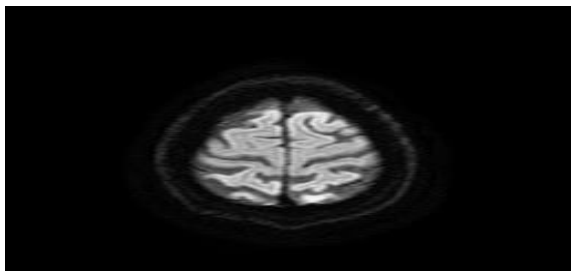

Fig 9: Test image 5

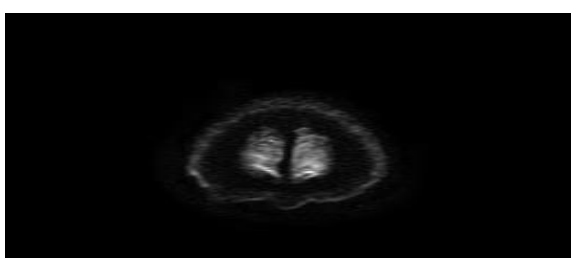

Fig 10: Test image 6

Table 1: Comparison between SVM and ANN

\begin{tabular}{|l|l|l|}
\hline Methods & $\begin{array}{l}\text { Correct } \\
\text { classification } \\
\text { percentage }\end{array}$ & $\begin{array}{l}\text { Incorrect } \\
\text { classification } \\
\text { Percentage }\end{array}$ \\
\hline SVM & $95.3 \%$ & $4.7 \%$ \\
\hline ANN & $58.33333 \%$ & $41.66777 \%$ \\
\hline
\end{tabular}




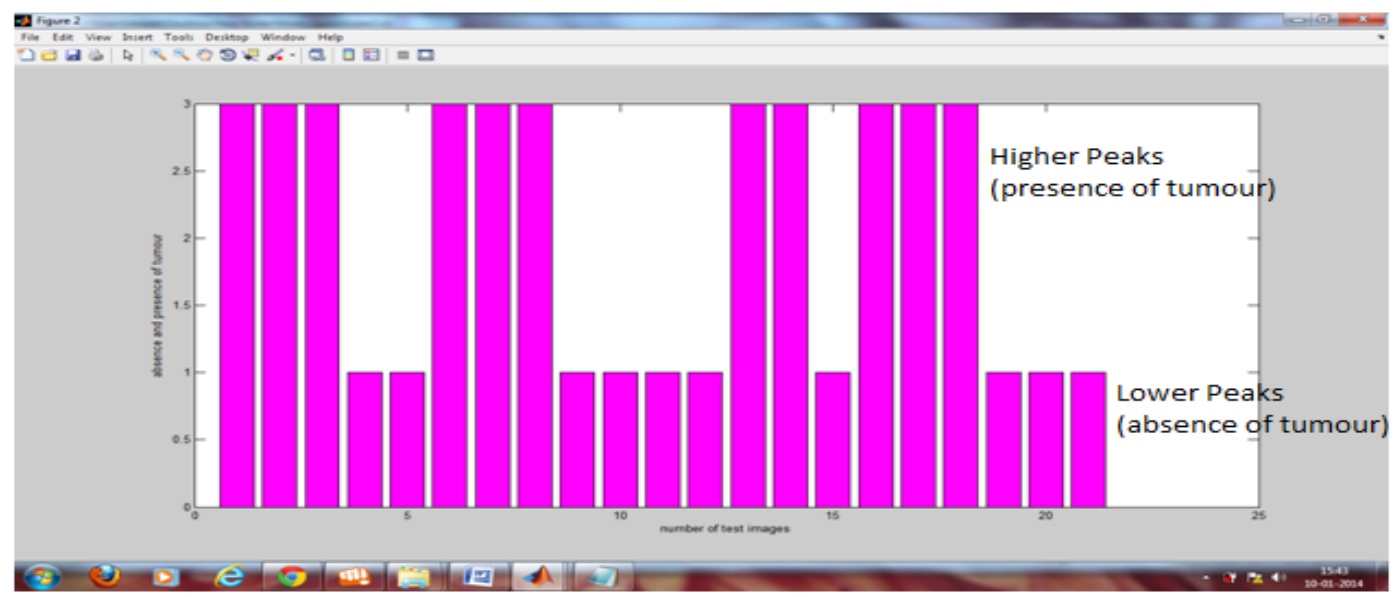

Fig 11: Presence and absence of tumour in different MR images using SVM

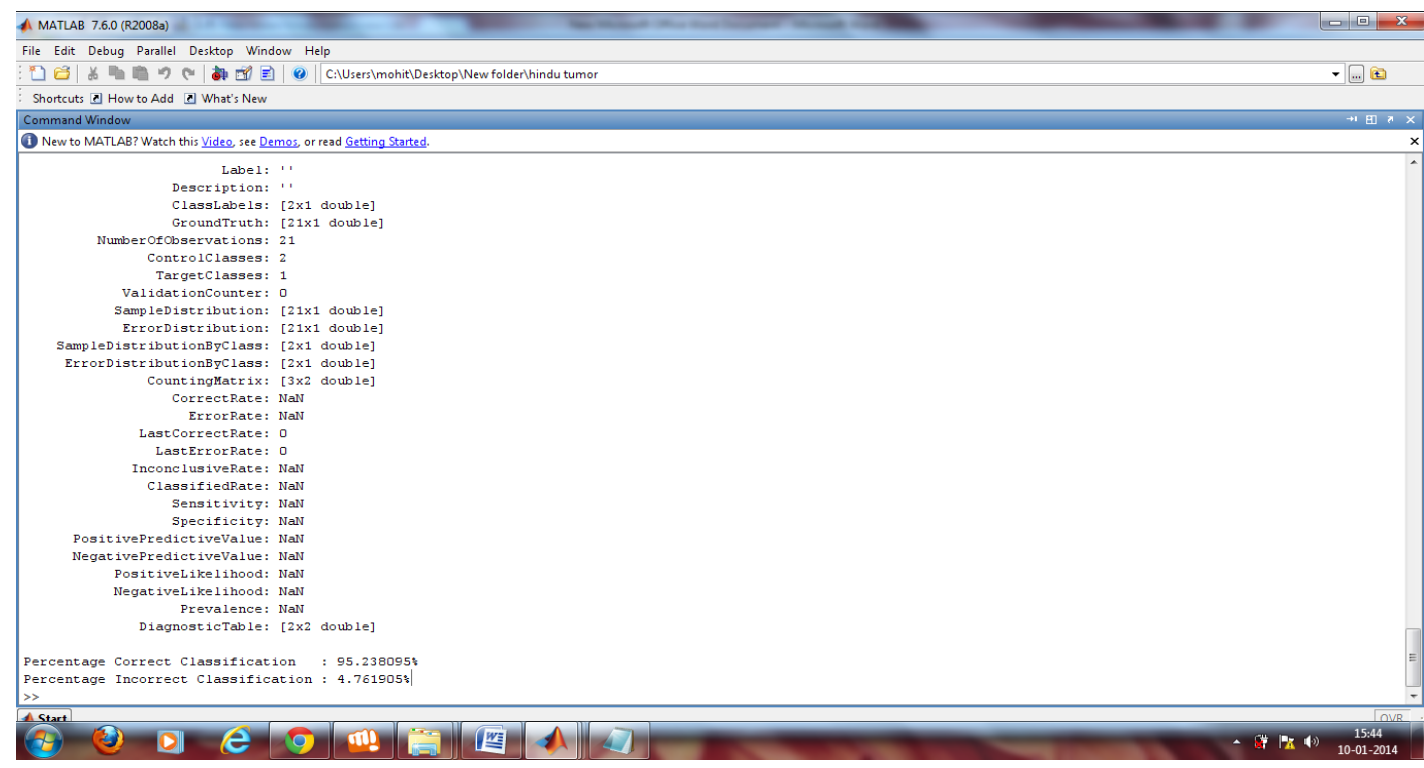

Fig 12: Snap-shot of MATLAB Command window for the results of SVM indicating correct and incorrect classification percentage 


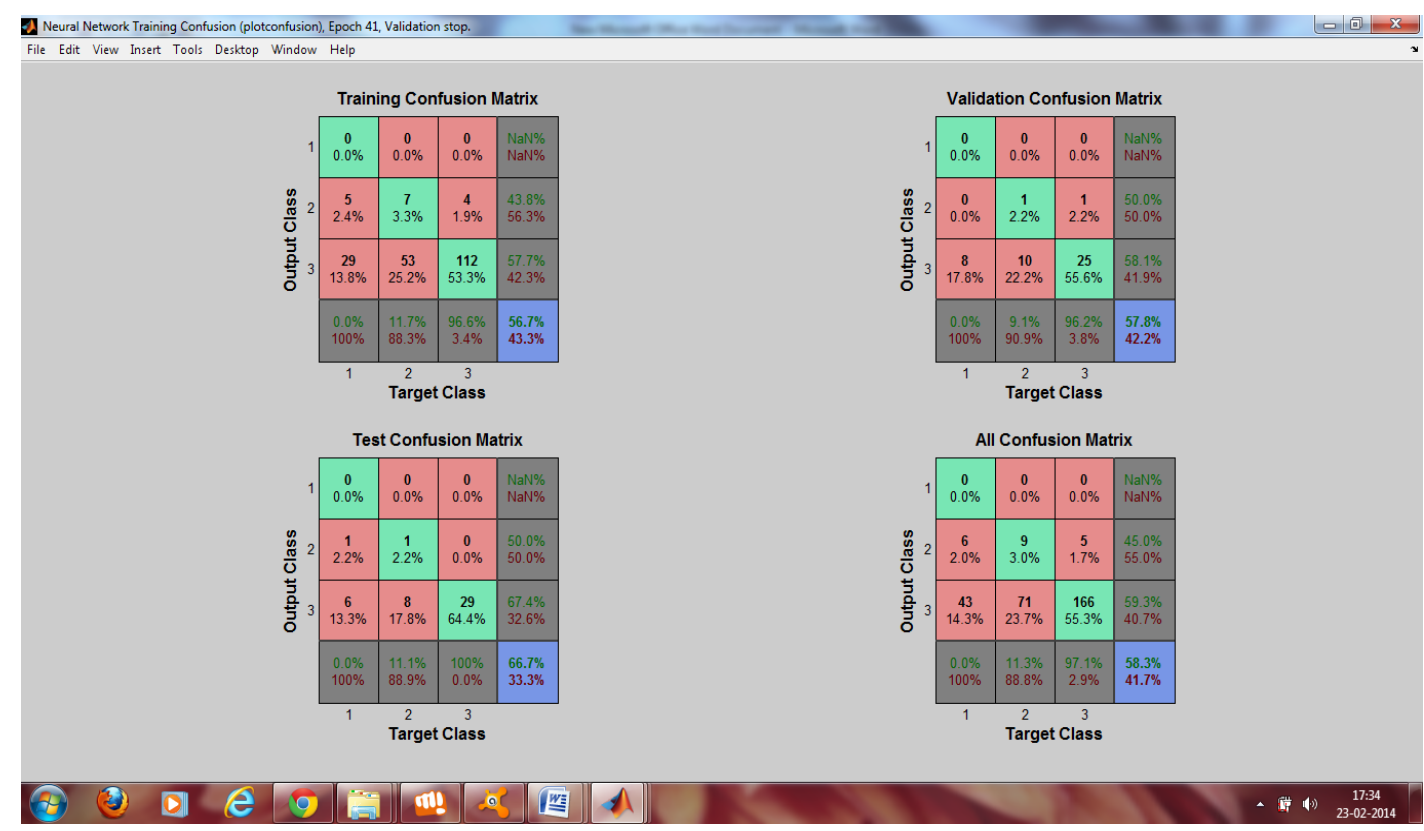

Fig 13: Snap-shot of MATLAB figure showing Training, Validation, Test and All Confusion matrixes for ANN

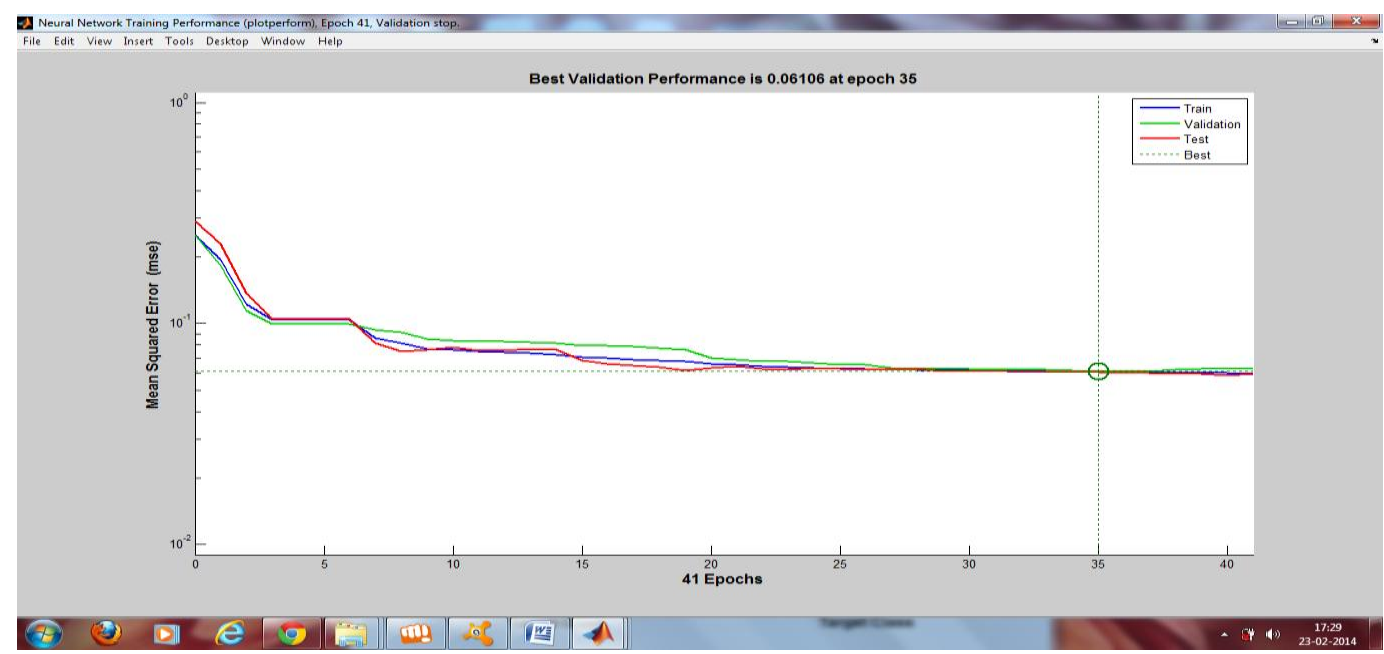

Fig 14: Snap-shot of MATALB figure of Validation Performance plot showing Mean Square Error at each epochs for ANN

\section{REFERENCES}

[1] M. Murugesan, R.Sukanesh, "Automated Detection of Brain Tumour in EEG Signals Using Artificial Neural Networks" 2009 International Conference on Advances in Computing, Control, and Telecommunication Technologies.

[2] Satish Chandra, Rajesh Bhat, Harinder Singh, "A PSO Based method for Detection of Brain Tumours from MRI” 978-1-4244-5612-3/09/\$26.00_c 2009 IEEE.

[3] M. Usman Akram, Anam Usman, "Computer Aided System for Brain Tumour Detection and Segmentation" 978-1-61284-941-6/11/\$26.00 @2011 IEEE.

[4] V.P.Gladis pushpa rathi, S.Palani, "Detection and Characterization of Brain Tumour Using Segmentation based on HSOM, Wavelet packet feature spaces and ANN" 978-1-4244-8679-3/11/\$26.00 @2011 IEEE.
[5] J.selvakumar, A.Lakshmi, T.Arivoli, "Brain Tumour Segmentation and Its Area Calculation in Brain MR Images using K-Mean Clustering and Fuzzy C-Mean Algorithm" IEEE-International Conference On Advances In Engineering, Science And Management (ICAESM 2012) March 30, 31, 2012.

[6] Rupsa Bhattacharjee, Monisha Chakraborty, "Brain Tumour Detection From MR Images: Image Processing, Slicing and PCA Based Reconstruction" 2012 Third International Conference on Emerging Applications of Information Technology (EAIT).

[7] T. Uchiyama, K. Mohri, M. Shnkai, A. Ohshma, H. Honda, T. Kobayashi, T. Wakabayashi and J. Yoshida, "Position sensing of magnetic gel Using MI Sensor for Brain Tumour Detection" IEEE Transaction on magnetics, Vol. 33, No. 5, September 1997. 
[8] C.C. Leung', W.F. Chen', P.C.K. Kwok3, and F.H. Y. Chan, "Brain Tumor Boundary Detection in MR Image with Generalized Fuzzy Operator"0-7803-7750-8/03/00 02003 IEEE.
[9] Yun Zhou, Sung-Cheng Huang, Shanglian Bao, Dean F. Wong, "Parametric Imaging and Statistical Mapping of Brain Tumor in Ga-68 EDTA Dynamic PET Studies" 07803-7324-3/02 @ 2002 IEEE. 